\title{
Werbung und/oder Offentlichkeitsarbeit in der Pfarrgemeinde?
}

\author{
von Karl Grüner
}

In immer mehr Pfarrgemeinderäten entstehen sog. Sachausschüsse für Öffentlichkeitsarbeit. Mit viel Mühe und noch mehr gutem Willen gestalten sie Pfarrblätter, malen Plakate, vervielfältigen Handzettel und schreiben Artikel für die Lokalpresse. Manchmal können sie sich über kleine Erfolge ihrer Arbeit freuen, wenn ein Bildungsabend, den sie angekündigt haben, gut besucht wurde oder wenn eine Kollekte, für idie sie geworben hatten, besser ausfiel als erwartet. Meist aber sind sie enttäuscht, erkennen ihr Unvermögen und rufen dann nach dem Werbefachmann, von dem sie erhoffen, daß er ihnen mit einigen Tips aus seiner Trickkiste auf die Beine helfen kann. Oder sie greifen nach Büchern und Arbeitshilfen, von denen inzwischen nicht wenige auf dem Markt sind. Dabei werden sie jedoch leicht durch unterschiedliche Begriffe verwirrt. Da ist von „Werbung"1 die Rede, von "Public Relations“" oder von "Offentlichkeitsarbeit"s. Und wenn sie ein wenig in diese Bücher hineinlesen, dann tauchen auch noch Ausdrücke wie „Propaganda“, „Reklame“, „Publicity“, "Information", "Image" usw. auf. So schreibt z. B. W. Wilckens in der Einführung zum „Praktikum kirchlicher Offentlichkeitsarbeit": „Wir sprechen abwechselnd vom Werben und von Public Relations. Und immer wieder kommt der Ausdruck Information vor. Aber ständig meinen wir - Offentlichkeitsdienst der Kirche oder der Gemeinde."

Die Verantwortlichen für Offentlichkeitsarbeit in den Pfarrgemeinderäten fragen sich zu Recht: Sind Werbung, Public Relations und Offentlichkeitsarbeit dasselbe und wenn nicht, womit von all dem sollen wir uns denn nun befassen?

Im folgenden soll eine Klärung und gegenseitige Abgrenzung der genannten Begriffe vorgenommen werden.

\section{Soll die Pfarrgemeinde Werbung treiben?}

Für eine Reihe von Autoren (vgl. Anm. 1) scheint es keine Frage zu sein, daß sich auch die Kirche der Werbung zu bedienen hat. Es ist jedoch nicht immer eindeutig, was sie unter Werbung verstehen. Eine der gängigen Definitionen lautet z. B.: „Werben heißt Nachricht geben in Willen bewegender Form." ${ }^{5}$ In diesem Sinne ist Werbung natürlich auch in der Kirche möglich. Von jeder Predigt und der Verkündigung überhaupt läßt sich sagen, daß sie eine (frohe) Nachricht ist, die den Willen (zur Umkehr, zur Nachfolge Christi, zur Nächstenliebe usw.) bewegen soll. Ist Verkündigung also Werbung? Manche bejahen diese Frage. Konsequenterweise müßten sie sich dann auch moderner Werbemethoden bedienen. Ansonsten könnte man ihnen vorwerfen, daß sie als "Werbemanager Gottes" angesichts der sich leerenden Kirchenbänke die "Sache Jesu" denkbar schlecht verkaufen ...

Wie dieses Beispiel zeigt, ist die genannte Definition viel zu verschwommen, um zu einer klaren Begriffsbestimmung zu taugen. Eindeutiger ist die Definition, die von der Deutschen Public-Relations-Gesellschaft (DPRG) vorgelegt wird. Sie versteht

Dipl.-Theologe Karl Grüner ist wissenschaftlicher Mitarbeiter am Lehrstuhl für Pastoraltheologie an der Gesamthochschule Eichstätt. 
unter Werbung „alle Maßnahmen, die auf die unmittelbare Auslösung eines Kaufentschlusses oder auf die Inanspruchnahme einer Dienstleistung abzielen"в.

Werbung will also verkaufen, sei es eine Ware oder eine Dienstleistung. In diesem Sinne ist sie uns von unserer täglichen Erfahrung her geläufig. Auf Schritt und Tritt, in Radio und Fernsehen, in Zeitungen und Illustrierten, von Plakatsäulen und Kinoleinwänden werden wir zum Kaufen und Konsumieren aufgefordert oder zur Inanspruchnahme von Dienstleistungen im weitesten Sinn (z. B. auch Besuch von Kino, Theater, Sport- oder Bildungsveranstaltungen).

Daß bei dem Versuch, einen Kaufentschluß auszulösen, mitunter auch unlautere Methoden zur Anwendung kommen, soll hier nur beiläufig erwähnt werden. Aber auch die scheinbar lauteren Werbemaßnahmen bestehen nicht selten aus psychologischen Appellen an das Unterbewußtsein, durch die die freie Entscheidungsfähigkeit des Menschen beeinträchtigt werden soll. Diese Methoden sind inzwischen so selbstverständlicher Bestandteil und sagar Gradmesser guter (sprich erfolgreicher) Werbung, daß sie bei der Frage nach dem Einsatz von Werbung in der Kirche und Gemeinde mit bedacht werden müssen.

Zunächst aber die Frage: Gibt es in unseren Pfarrgemeinden überhaupt Situationen und Anlässe, bei denen Werbung im Sinne der genannten Definition angebracht ist?

Es mag pseudomoderne Pfarrer geben, die den "Weißen Riesen" Jesus als den "großen Saubermacher" anpreisen, der den "Grauschleier" von unserer Seele wegwäscht und in unserem Innersten für das "strahlendste Weiß unseres Lebens" sorgt.

Von einem solchen Zerrbild abgesehen kann man durchaus im Gemeindeleben Gelegenheiten finden, in denen etwas verkauft oder eine Dienstleistung in $\mathrm{An}_{\mathrm{n}}$ spruch genommen werden soll: Pfarrfeste, Basare, Tombolen, deren Erlöse guten Zwecken dienen, Dritte-Welt-Aktionen, Kurse, Vorträge usw. Wären hierfür nicht Maßnahmen angebracht, die auf die „unmittelbare Auslösung eines Kaufentschlusses" zur Unterstützung der guten Sache oder auf die "Inanspruchnahme einer Dienstleistung" im Interesse der eigenen Fortbildung abzielen?

Vordergründig gesehen: ja. Wenn es den Veranstaltern ausschließlich um die finanzielle Seite geht, mag ein Werbefeldzug mit entsprechend eingesetzten Medien und zugkräftigen Gags das Richtige sein und den gewünschten Erfolg bringen. Wann aber ist diese Situation in einer Pfarrgemeinde wirklich gegeben?

Aktionen für die Dritte Welt oder die Mission zielen nicht in erster Linie auf ein möglichst hohes Spendenaufkommen, so not-wendig dieses sein kann, sondern darauf, christliche Solidarität und Partnerschaft mit Armen, Kranken, Ausgebeuteten und Unterdrückten zu üben, Vorurteile abzubauen usw. Dasselbe gilt für Spendenaktionen zugunsten eines Altenheims, einer beschützenden Werkstatt oder ähnlicher sozialer Einrichtungen. Im Zentrum steht dabei nicht (oder sollte jedenfalls nicht stehen!) die „Auslösung eines Spendenentschlusses“, sondern die Information über die betroffenen Menschen, ihre Nöte und Probleme. Werbemaßnahmen sind hierfür nicht das geeignete Mittel.

Bildungsveranstaltungen in der Pfarrgemeinde könnte man als Dienstleistungen sehen, für deren Inanspruchnahme Werbung im Sinne der Definition angebracht wäre. Wenn durch eifriges Rühren der Werbetrommel der Saal voll wird, dürfte der beabsichtigte $Z$ weck doch erfüllt sein!? 
Auch das ist eine vordergründige Sicht. Erwachsenenbildung in den Pfarrgemeinden wird noch immer von der Magie der großen Zahl beherrscht. 60 Teilnehmer : gelungener Abend; 10 Teilnehmer : ein Mißerfolg! Daß jedoch ein Kreis von 10 Teilnehmern eine wesentlich bessere Voraussetzung für Lernerfolge bietet als ein voller Saal mit 60 Leuten, ist auf der gemeindlichen Ebene noch zu wenig erkannt worden. Dies würde natürlich eine völlige Umorientierung der bisherigen Bildungsarbeit in den Pfarrgemeinden erfordern, z. B. weitgehenden Verzicht auf einzelne Vorträge, deren Themen am zufälligen örtlichen oder diözesan vermittelten Referentenangebot orientiert sind. Wenn Bildungsarbeit ein Teil der Gemeindepastoral ist, dann sollte sie auch in die Gesamtpastoral der Gemeinde eingebettet sein. Das bedeutet, daß ein Thema nicht isoliert von den jeweiligen Bedürfnissen der Gemeinde gewählt wird, sondern daß es schon vorher als Problem oder Anliegen in der Gemeinde vorhanden sein oder geweckt werden sollte. Das erfordert kontinuierliche Arbeit an einem Thema (z. B. einer Jahreslosung), wofür sich eher die Form von Arbeitskreisen anbietet als der Einzelvortrag, der jedoch als ergänzendes Angebot nicht völlig auszuschließen ist.

Es leuchtet ein, daß bei einer solchen, in die Gesamtpastoral der Gemeinde eingebauten Bildungsarbeit nicht in erster Linie Werbung geboten ist, wie sie ohne weiteres bei der Veranstaltung eines heimatkundlichen Lichtbildervortrages angebracht sein kann. Vielmehr muß hier, wie auch in den vorgenannten Beispielen (Spendenaktionen für Dritte Welt, Altenheime usw.) eine umfassende Offentlichkeitsarbeit einsetzen.

\section{Offentlibhkeitsarbeit der Pfarrgemeinde}

Worin liegt der Unterschied zur Werbung?

Der Offentlichkeitsarbeit geht es nicht um die unmittelbare Auslösung eines Kaufentschlusses, auch wenn sie diesen nicht völlig ausschließen will. Offentlichkeitsarbeit will ein allgemeines Klima des Vertrauens schaffen, eine Atmosphäre, die Kommunikation ermöglicht.

Offentlichkeitsarbeit ist auch mehr als Public Relations, obwohl sie oft damit gleichgesetzt wird. Public Relations wird definiert als „Meinungspflege zur Verstärkung eines positiven Images " . Das bedeutet, daß alles, was nicht zu einem positiven Image in der Offentlichkeit paßt, verschwiegen und versteckt werden müßte. Man dürfte nur die Schokoladenseite der Pfarrgemeinde in der Offentlichkeit wahrnehmen.

Offentlichkeitsarbeit will jedoch nichts verschweigen. Sie will ehrlich und offen informieren. Sie will Gemeinde nach innen und außen durchsichtig machen. Es geht ihr nicht darum, durch Herauskehren positiver Eigenschaften die Kritiker zum Schweigen zu bringen. Sie versucht vielmehr, mit den Kritikern ins Gespräch zu kommen, sie $z u$ überzeugen oder sich von ihnen überzeugen zu lassen. Offentlichkeitsarbeit hat also in erster Linie mit Kommunikation zu tun. Man könnte definieren: Öffentlichkeitsarbeit ist die systematische Organisation des Gesprächs in der Pfarrgemeinde und ïber sie binaus.

Werbung ist erfolgsorientiert. Offentlichkeitsarbeit ist partnerorientiert. Werbung versucht zu überreden, manchmal auch zu überrumpeln; Offentlichkeitsarbeit sucht das Gespräch und die Mitbeteiligung des Partners. Marktschreierische Ubertreibungen sind ihr ebenso fremd wie psychologische Appelle an das Unterbewußtsein, durch die die menschliche Entscheidungsf reiheit ausgeschaltet werden soll.

Wie kann die Offentlichkeitsarbeit der Pfarrgemeinde konkret aussehen? 
1. Die Offentlichkeitsarbeit führt kein Eigenleben; sie ist nicht Selbstzweck, sondern steht im Dienste der Gesamtpastoral einer Pfarrgemeinde. Ohne pastorales Planungskonzept, z. B. im Zusammenhang mit der Bearbeitung eines Jahresthemas ${ }^{8}$, wird sich auch die Offentlichkeitsarbeit, ebenso wie die Seelsorge, in zusammenhanglose Einzelaktivitäten verlieren und die ihr eigenen Möglichkeiten kaum optimal nutzen können. Wird jedoch ein pastorales Konzept mit phantasievollen Maßnahmen der Offentlichkeitsarbeit den Gemeindemitgliedern nahegebracht, so sind davon mit Sicherheit neue Impulse für das Gemeindeleben zu erwarten. Eine partnerorientierte und damit auch gesprächsermöglichende Offentlichkeitsarbeit wird nämlich in der Regel auch aus der Gemeinde eine Rückkopplung erfahren, die wieder neue Anregungen für die weitere pastorale Planung enthält.

2. Dies zielt bereits auf die Erkenntnis, daß pastorales Handeln heute ein Geschehen der ganzen Gemeinde sein soll. Die Offentlichkeitsarbeit müßte dies mit den ihr zur Verfügung stehenden Mitteln

a) durch Information noch mehr bewußt machen und

b) erst richtig ermöglichen, indem sie die für die Verwirklichung gemeinsamen Handelns notwendige Kommunikationsbasis bereitstellt.

Konkret heißt das, daß die Verantwortlichen für Offentlichkeitsarbeit Sorge tragen müssen,

- daß alle das Gemeindeleben betreffenden Informationen öffentlich zugänglich sind,

- daß Einzelne und Gruppen, bzw. deren Repräsentanten, Gelegenheit erhalten, ihre Meinung öffentlich zu äußern,

- daß auch diejenigen, die sich selbst nicht so gut ausdrücken können, mit ihrer Meinung zu Wort kommen,

- daß die distanziert kirchlichen Gemeindemitglieder durch speziell für sie bestimmte Informationen in das Gespräch der Gemeinde miteinbezogen werden ${ }^{\theta}$.

Das erfordert eine gute Kenntnis der Gemeinde sowie zahlreiche persönliche Kontakte der Verantwortlichen für Offentlichkeitsarbeit mit den Gemeindemitgliedern und der Gemeindeleitung. Und es erfordert die Fähigkeit, verschiedene Medien zum jeweils richtigen Anlaß einzusetzen (z. B. Kontaktbriefe, Handzettel, Schaukasten, Plakate, Lokalzeitung, Pfarrblatt u. a.). Zentrale Bedeutung kommt dabei dem Pfarrblatt zu, das als "technisch enmöglichter Versammlungsraum "10 die ganze Gemeinde gleichsam um einen „runden Tisch“ versammelt ${ }^{11}$ und alle zu Wort kommen läßt, die etwas zu sagen haben.

3. Voraussetzung für dieses Gespräch der Gemeinde ist die Bereitschaft zur Information von beiden Seiten: Die Gemeindeleitung muß über Meinungen und Vorgänge innerhalb der Gemeinde unterrichtet werden, und sie darf ihrerseits keine wichtigen Informationen zurückhalten. Sie käme sonst wieder in Gefahr, autoritäres Herrschaftswissen zu speichern, um die Gemeinde in Unmündigkeit zu halten. Dies gilt nicht nur für den Pfarrer, sondern besonders auch für den Pfarrgemeinderat. Es kommt nicht selten vor, daß Pfarrgemeinderäte über die Köpfe ihrer Gemeinde hinweg „regieren“, weil sie alle Informationen, die zu Entscheidungen führen, für sich behalten.

Es müßte Aufgabe der Offentlichkeitsarbeit sein, die Gemeinde über bevorstehende Entscheidungen des Pfarrgemeinderats bereits in einem so frühen Stadium zu unter- 
richten, daß eine edhte Mitsprache und Mitwirkung bei der Entscheidungsfindung möglich ist. Erfolgt die Information erst nach der Beschlußfassung, wird die Gemeinde wieder zum bloßen ausführenden Organ degradiert.

Ein Beispiel aus einer Gemeinde der Erzdiözese Köln mag verdeutlichen, wie eine solche Offentlichkeitsarbeit aussehen kann. Es ging um die Einführung der neuen Taufliturgie in der Pfarrgemeinde. Sie war für den 1. Januar 1972 vorgesehen. Im Juli 1971, also ein halbes Jahr vorher, veröffentlichte der Pfarrer folgende drei Thesen im Pfarrblatt und bat um Stellungnahme dazu: „1. Die Kirche handelt unverantwortlich, wenn sie die Taufe weiter so spendet wie bisher. Vor der Taufspendung muß sichergestellt werden, daß die Eltern des Kindes gläubige Menschen sind, die dafür garantieren, daß dieses Kind wirklich zum Glauben geführt wird. 2. Die Kirche kann nur noch da taufen, wo auch christliche Gemeinden sind, die nicht in ihrem religiösen Leben erschlafft sind. Wenn getauft wird, muß die Gemeinde auch Gewähr dafür bieten, daß der so in ihre Gemeinschaft Aufgenommene Glaubenskraft und Vorbild und christliche Gemeinschaft erfährt. 3. Die Taufe ist für den Menschen so lebensentscheidend, $\mathrm{da} ß$ sie schon im frühesten Lebensalter gespendet werden muß. Aber die beiden oben angegebenen Bedingungen sind Voraussetzung für die Möglichkeit der Taufspendung überhaupt."

Im nächsten Pfarrblatt, das im September erschien, war ein Leserbrief, der gegen die Thesen des Pfarrers Stellung bezog. Seine Quintessenz: Die Thesen widersprechen dem Wesen des Christentums, zumindest in der Form, in der es bisher gelehrt und praktiziert wurde, und sie sind in der Praxis nicht realisierbar. An den Leserbrief schloß sich als erste Information über die bevorstehende Änderung ein Ausschnitt aus den "Vorbemerkungen zur erneuerten Taufliturgie für die deutschen Diözesen" an. Im Oktoberheft erschien ein (fiktives) Gespräch zwischen einem Priester, einer Frau Schmitz und einem Herrn Müller, die die in der Gemeinde inzwischen laut gewordenen Standpunkte aufgriffen und miteinander diskutierten. Im November folgten weitere Auszüge aus den "Vorbemerkungen“. Gleichzeitig fand an zwei Abenden ein Glaubensseminar statt, das den neuen Taufritus zum Thema hatte. Im Dezemberheft schließlich erläuterte der Pfarrer noch einmal die neue Form der Taufliturgie und kündigte ihre Einführung zum 1. Januar an. Außerdem bedankte er sich dafür, daß sich so viele Gemeindemitglieder am Gespräch über die neue Taufliturgie beteiligt hatten.

Man hätte es auch anders machen können: die neuen Richtlinien veröffentlichen oder in der Kirche verlesen, den Termin bestimmen und einführen. Oder man hätte auf Plakaten, Flugblättern oder im Pfarrblatt "werben “ können, indem man die Vorzüge der neuen Liturgie angepriesen hätte. In beiden Fällen wäre der Wichtigste, nämlich der betroffene mündige Gemeindechrist, bei der Einführung einer bedeutenden Neuerung nicht beteiligt worden.

Die Offentlichkeitsarbeit der angeführten Gemeinde bestand im wesentlichen darin, daß ein Problem mit Hilfe des Mediums Pfarrblatt ins öffentliche Gespräch gebracht und auf diese Weise Verständnis und Bereitschaft zur Annahme einer Neuerung in der Gemeinde geweckt wurde (auch wenn aus kirchendisziplinären Gründen die Möglichkeit einer Ablehnung durch die Gemeinde nicht bestanden hat).

4. Alle die bisher aufgeführten Aufgaben und Möglichkeiten der Offentlichkeitsarbeit sind auf das Innenleben der Gemeinde bezogen. Christliche Gemeinden sind jedoch keine Gettos. Sie sind zwar, jede für sich, voll und ganz Kirche, eben die 
Ekklesia am Ort. Dennoch sind sie auch eingebettet in das Gesamt der diözesanen Ortskirche und in die Weltkirche. Die Offentlichkeitsarbeit der Gemeinde muß auch diese überörtlichen Bezüge berücksichtigen und beispielsweise Informationen aus der Welt- und Diözesankirche, die für das Gemeindeleben relevant sind, in geeigneter Form in das Gespräch der Gemeinde einbringen. Dazu gehören z. B. auch Kontakte mit Gemeinden in Missionsländern.

Umgekehrt können Nachrichten aus der Gemeinde, z. B. Berichte über gelungene Aktionen für andere Gemeinden ebenso interessant sein und sollten deshalb auch der Bistumszeitung zur Veröffentlichung angeboten werden.

Neben den innerkirchlichen Bezügen hat jede Pfarrgemeinde auch eine Affinität zu ihrer gesellschaftlichen Umwelt. Es ist ja ihr Auftrag, "Licht der Welt" und „Salz der Erde $^{\text {“ }}$ zu sein. Das bedeutet, daß die Anliegen und Aufgaben der Gesellschaft, insbesondere des unmittelbaren kommunalen Umfeldes, in der Gemeinde immer wieder bewußt gemacht werden sollen (Pfarrblatt, Schaukasten u. ä.), um dann unter Umständen sogar in konkrete Aktionen einzumünden.

Aber die gesellschaftliche Offentlichkeit außerhalb der Pfarrgemeinde sollte auch einen Einblick in das Geschehen innerhalb der Gemeinde erhalten - wie könnte letztere sonst ihrem Auftrag, Zeichen des Heils zu sein und der Welt Zeugnis zu geben, gerecht werden?

Das Medium, das sich hierfür anbietet, ist im allgemeinen die lokale Presse, zu der die Verantwortlichen für Offentlichkeitsarbeit regelmäßige und gute Beziehungen unterhalten sollten.

\section{Zusammenfassung}

Es dürfte klar geworden sein, daß die besondere Situation einer Pfarrgemeinde mehr erfordert als gelegentliche oder auch regelmäßige Werbemaßnahmen. Das, was Werbung im Sinne der oben verwendeten Definition erreichen kann, sind vordergründige Ergebnisse, wie z. B. höheres Spendenaufkommen oder ein gut besuchter Vortrag. Da, wo es in erster Linie um solche Erfolge geht, sind Werbemaßnahmen, soweit es sich nicht um unlautere, die freie Entscheidung beeinträchtigende Methoden handelt, durchaus angebracht.

$\mathrm{Da}$ sich aber die Gemeindepastoral nicht mit so vordergründigen Ergebnissen zufriedengeben kann, ist eine umfangreiche Offentlichkeitsarbeit vonnöten. Diese ist wiederum von Public Relations zu unterscheiden, denen es um ein möglichst gutes Image geht, weshalb nur die positiven Seiten einer Sache herausgestellt werden. Hofberichterstattung ist jedoch keine Offentlichkeitsarbeit. Es geht vielmehr darum, die Gemeinde „ins Gespräch zu bringen“, d. h. die Kommunikation in der Gemeindc $\mathrm{zu}$ fördern, ja regelrecht $\mathrm{zu}$ organisieren und ebenso das Gespräch $\ddot{u} b e r$ die Gemeinde binaus, damit diese als Kirche am Ort ihrem Auftrag, "Zeichen und Werkzeug des Heils" zu sein, so gut wie möglich gerecht werden kann.

\section{Anmerkungen:}

1. F. U. Gass: Werbung im Dienst der Kirche, Limburg 1966; H. Kauber: Gewußt wie! Tips für die Werbearbeit in der Kirche, Gelnhausen und Berlin 1966; M. Schütte: 
Kirchliche Werbung - Aufgaben, Ziele, Möglichkeiten, Düsseldorf 1966; W. Wilken: Die Werbung der Kirche, Berlin ${ }^{2} 1970$.

2. J. Scharrer: Public Relations und Seelsorge, in: K. Rahner / B. Häring: Wort in Welt, Bergen-Enkheim 1968, 344-352; W. Wilken: Brücken zur Kirche - Public Relations der Kirche, Berlin 1967.

3. Offentlichkeitsarbeit der Pfarrgemeinde. System-Methoden-Praxis, hrsg. v. Osterreichischen Pastoralinstitut, Wien-Freiburg-Basel 1973; Offentlichkeitsarbeit in der Diakonie. Ein Handbuch für die Praxis, Stuttgart 1969; H. Wagner / K. Grüner / E. Bieger: Offentlichkeitsarbeit der Pfarrgemeinde. Der Ausschuß für Offentlichkeitsarbeit im Pfarrgemeinderat. Reihe "medienpraxis“, Praktische Medienarbeit 1, hrsg. v. der Kirchlichen Zentralstelle für Medien, Referat Kommunikationspädagogik, Bonn 21976; W. Wilken: Praktikum kirchlicher Offentlichkeitsarbeit. Kampagnen Analysen Modelle, Hamburg 1972.

4. Wilken, Praktikum 15.

5. Nach Weidenmüller, zit. bei Gass: Werbung 28.

6. J. Scharrer / E. Löcher: Kontakte oder Konflikte? Seelsorge vor der Entscheidung, Frankfurt 1970, 12.

7. Nach Gass: Werbung 30.

8. Vgl. W. Friedberger: Pastorale Planung. Handreichungen zur Planung der Seelsorge in der Pfarrei, Würzburg 1976; und G. Reichert: Arbeit mit einem Jahresthema als Möglichkeit der Pastoralplanung in der Pfarrgemeinde, in: „Lebendige Seelsorge“, 27:1976, $336-339$.

9. K. Grüner: Die Teilnahme am Gespräch der Kirche, in: „Diakonia“, 9:1978, $182 \mathrm{f}$.

10. H. Wagner: Der Pfarrbrief. Fernkurs für Pfarrbriefredakteure (und solche, die es werden wollen), in: "Lebendige Zelle“, Heft 2, 1974, 72.

11. Pfarrblatt. Instrument der Gemeinde-Kommunikation. Reihe "medienpraxis“, Praktische Medienarbeit 2, hrsg. v. der Zentralstelle Medien, Referat Kommunikationspädagogik, Bonn 1976, 2 f.

\section{S UM M A R Y}

Advertising which aims at attracting immediate custom or services might lead to superficial results, such as an increase of funds, but this can never be sufficient motive for the pastoral work of the parish. A comprehensive Public Relations work seems to be needed, one not involved merely in fostering an image. Public Relations for Pastoral Work means the systematic organisation of communications within and beyond the parish boundaries. Differing from advertising, it aims at fostering dialogue and participation by partners. Pastoral Public Relations strives to make all information about the parish really available to parishoners, and those who have something to say should be given the opportunity of doing so. Non-practicing members of the parish should be invited to share by items of interest specifically bearing them in mind.

\section{RESUME}

La publicité et/ou le travail public dans les communautés paroissiales, plus précisément dans les conseils des communautés paroissiales, sont l'abjet d'une conception différente parce que les notions sont pour longtemps imprécises et chatoyantes. L'auteur essaie d'apporter un peu 
de clarté et montre ensuite à quoi le travail public de la communauté paroissiale peut ressembler, de façon concrête. La situation exige plus que des mesures publicitaires occasionnelles ou même régulières, mais précisément un vaste travail public qui doit étre très bien différencié par les services de relations avec le public. Ce qui est nécessaire n'est en aucun cas de soigner son aspect dans le sens des relations publiques. Le but est plutôt de "faire entrer" la communauté "dans la conversation“, de promouvoir la communication à l'intérieur de la communauté et même d'étendre le dialogue au-delà de la communauté afin que l'Eglise puisse remplir sa mission sur place, même la remplir de son mieux.

\section{RESUMEN}

La propaganda y el trabajo de cara a la opinión pública en las parroquias, mejor dicho en los consejos parroquiales, son objeto de interpretaciones diversas porque los conceptos son ámpliamente imprecisos y poco diáfanos. El autor trata de aportar claridad y muestra como podría presentarse en concreto la actividad de los consejos parroquiales frente a la opinión pública. La situación exige más que esporádicas e incluso periódicas acciones de propaganda, es decir un ámplio trabajo de divulgación que se diferencia perfectamente de las Public Relations. No es necesario cuidar la fisonomía al modo como lo hacen las Public Relations. La meta es, por el contrario, „introducir el diálogo" en la parroquia, promover la comunicación social en la comunidad, al mismo tiempo que el diálogo sobre la parroquia, para que la Iglesia pueda cumplir su cometido en caida lugar, lo mejor posible. 